\title{
SIMPLIFICATION OF THE WHITEHEAD-HUNTING- TON SET OF POSTULATES FOR THE ALGEBRA OF LOGIC
}

\author{
BY A. H. DIAMOND*
}

1. Introduction. In 1916, B. A. Bernstein $\dagger$ obtained a reduction in the number of postulates of the Whitehead-Huntington set $\ddagger$ for the algebra of logic. In this paper I secure a further reduction in the number of the postulates by combining the commutative and distributive laws and then proceed to investigate the postulates which result when the two reductions are effected at once.

I prove the sufficiency of the new postulates for the algebra of logic by showing that the original set can be derived from them. Finally I establish the independence of the new postulates by furnishing proof-systems of a simple arithmetic type.

2. The New Postulates. The new postulates have as undefined ideas the undefined ideas of the original set, namely, a class $K$ and two binary operations + and $\times$. The postulates are the Postulates 1-6 listed below. In Postulates 3 and 4 is implied the condition if the elements involved and their indicated combinations belong to $K$.

Postulate 1. $a+b$ is in $K$ whenever $a$ and $b$ are in $K$.

Postulate 2. $a b$ is in $K$ whenever $a$ and $b$ are in $K$.

Postulate 3. $b c+a=(c+a)(b+a)$.

Postulate 4. $(b+c) a=c a+b a$.

Postulate 5. For any element $b$ in $K$ there exists an element $b^{\prime}$ such that, whatever $a$ is, $a+b b^{\prime}=a$ and $a\left(b+b^{\prime}\right)=a$.

Postulate 6. There are at least two elements, $a$ and $b$, in $K$ such that $a \neq b$.

3. Sufficiency of the Postulates. Derivation of the WhiteheadHuntington Postulates. The Whitehead-Huntington postulates leave undefined a class $K$ and two binary operations + and $X$,

* American Field Service Fellow.

† See this Bulletin, vol. 22 (1916), pp. 458-459.

$\ddagger$ See the Transactions of this Society, vol. 5 (1904), pp. 288-309. The set referred to is the first of the three sets which occur in Huntington's paper. 
and are the Postulates Ia, Ib, . . , VI below. In Postulates IIIa-IVb is implied the condition if the elements involved and their indicated combinations belong to $\mathrm{K}$, and in $\mathrm{V}$ is implied the condition if the elements $z$ and $u$ of IIa and IIb exist and are unique.

Postulate Ia. $a+b$ is in $K$ whenever $a$ and $b$ are in $K$.

Postulate $\mathrm{Ib}$. $a b$ is in $K$ whenever $a$ and $b$ are in $K$.

Postulate IIa. There is an element $z$ such that $a+z=a$ for every element $a$.

Postulate IIb. There is an element $u$ such that $a u=a$ for every element $a$.

Postulate IIIa. $a+b=b+a$.

Postulate IIIb. $a b=b a$.

Postulate IVa. $a+b c=(a+b)(a+c)$.

Postulate IVb. $a(b+c)=a b+a c$.

Postulate V. For every element a there is an element $a^{\prime}$ such that $a+a^{\prime}=u$ and $a a^{\prime}=z$.

Postulate VI. There are at least two elements, $a$ and $b$, in $K$ such that $a \neq b$.

It is seen that Postulates 1, 2, 6 are precisely the Postulates Ia, Ib, VI, respectively, of the original set. Further, Bernstein has shown* that IIa, IIb, V of the original set follow from Postulates 1,2, and 5. Consequently, in order to prove the sufficiency of Postulates 1-6 for the algebra of logic, it remains to derive from the postulates 1-6 Postulates IIIa-IVb of the original set. In the proofs I give below I assume, in accordance with Bernstein's results, that IIa and IIb have already been proved.

ProOF OF IIIa. In 4 put $a=u$, using 1, 2, and IIb.

Proof OF IIIb. In 3 put $a=z$, using 1, 2, and IIa.

Proof of IVa. By 1, 2, 3, IIIa, and IIIb.

Proof of IVb. By 1, 2, 4, IIIa, and IIIb.

It can easily be verified that Postulates 3 and 4 can be derived from IIIa-IVb. It thus becomes apparent, when one also takes into account the results obtained by Bernstein, that Postulates 1-6 are equivalent to Postulates Ia-VI of the original set.

4. Independence of the Postulates. I establish the independence of Postulates 1-6 by giving the following examples of proof-

* See this Bulletin, loc. cit. 
systems, each of which violates the like-numbered postulate and satisfies all the other postulates of the set. These examples are all arithmetic systems, the elements being the numbers 0 and 1 . The symbol $f(a, b)(\bmod 2)$ in an arithmetic system denotes the least positive residue modulo 2 obtained from $f(a, b)$ by rejecting multiples of 2 . The operations + and $X$ are to be interpreted as the operations of ordinary arithmetic when they occur in the modular expression, otherwise they are to be interpreted as logical addition and logical multiplication.

\begin{tabular}{|c|c|c|c|}
\hline EXAMPLE & $K$ & $a+b$ & $a b$ \\
\hline 1 & 0,1 & $a+b+0 / a b+1(\bmod 2)$ & $a b(\bmod 2)$ \\
2 & 0,1 & $a b(\bmod 2)$ & $a+b+0 / a b+1(\bmod 2)$ \\
3 & 0,1 & $a+b(\bmod 2)$ & $a b(\bmod 2)$ \\
4 & 0,1 & $a b(\bmod 2)$ & $a+b(\bmod 2)$ \\
5 & 0,1 & $a b(\bmod 2)$ & $a b(\bmod 2)$ \\
6 & Null & & \\
\hline
\end{tabular}

Paris, France

\section{ON ANALYTIC FUNCTIONS WITH POSITIVE IMAGINARY PARTS}

BY J. L. DOOB* AND B. O. KOOPMAN

The purpose of this paper is to give an integral representation of a function analytic in a half-plane, and with positive imaginary part there. This can be used to obtain in a simple way the well known analytic representation of the resolvent of a selfadjoint transformation in abstract Hilbert space.

Theorem. Let $\phi(l)$ be a function analytic for $\Im(l)>0$. If

$$
\Im[\phi(l)] \geqq 0, \quad \quad \quad \limsup |t \Im[\phi(i t)]|<\infty,
$$

for $t$ real, $t>0$, then there is a uniquely determined monotone non-decreasing function $\alpha(\lambda)$, defined for $-\infty<\lambda<\infty$, satisfying

* National Research Fellow.

$\dagger$ If $\xi$ is any complex number, $\Re(\xi)$ and $\Im(\xi)$ will be used to denote its real and imaginary parts, and $\bar{\xi}$ its conjugate. 